\title{
Consentimiento informado en la nueva ley de derechos de los pacientes
}

\author{
RODOLFO FIGUEROA G. ${ }^{\mathrm{a}}$
}

\section{Informed consent in the patients' rights law}

The new regulation of patients' rights creates the right to informed consent, which allows accepting or refusing any medical treatment. Also, the patient has the right to be appropriately informed about a variety of aspects determined by the law. Patient's autonomy has limits. Artificial acceleration of death, euthanasia or assisted suicide are not permitted. The problem is that the law does not define those situations. The law provides the intervention of Ethics Committees when the doctor considers that the patient exposes himself to severe harm or the risk of death which would be avoided. This intervention impinges on patient's autonomy. Patients have the right to request discharge and medical facilities could discharge patients against their will if they do not accept medical recommendations. These limitations on autonomy should be explained because the law apparently makes the distinction between killing, letting die and the Double Effect Doctrine. There is plenty of literature questioning the validity of both. The law fails to regulate part of the medical practice, regarding life and death decisions. A lack of consensus could explain this omission. Doctors have a right to conscientious objection to some patient's requests.

(Rev Med Chile 2012; 140: 1347-1351).

Key words: Ethics Committees; Euthanasia; Informed Consent.

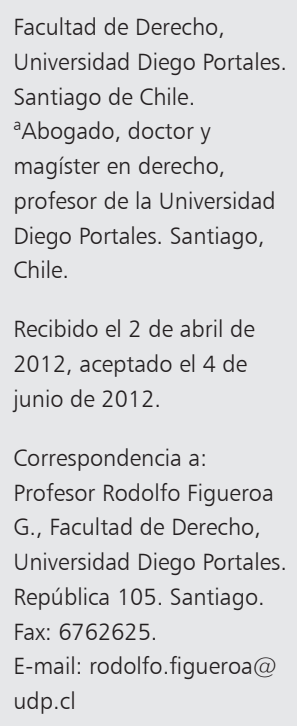

$\mathrm{E}$ 124 de abril de 2012 se publicó en el Diario Oficial la ley No 20.584 que regula los derechos y deberes de los pacientes. Sin embargo, esta ley entrará en vigencia el 1 de octubre de $2012^{1}$. Esta ley regula el consentimiento informado del paciente, proporcionándole autonomía para decidir a qué procedimientos médicos pueda someterse. La ley regula también el derecho a obtener la información necesaria para poder prestar el consentimiento. Junto con ello, la ley establece limitaciones a esta autonomía, prohibiendo la aceleración artificial de la muerte, la eutanasia y el auxilio al suicidio. En este trabajo se explica la normativa sobre el consentimiento informado y se discuten los límites al mismo.

\section{Regulación legal del consentimiento informado}

1. El consentimiento informado de los pacientes 1.1. La norma general

La ley reconoce expresamente en el artículo
14 el derecho de los pacientes al consentimiento informado:

"Toda persona tiene derecho a otorgar o denegar su voluntad para someterse a cualquier procedimiento o tratamiento vinculado a su atención de salud, con las limitaciones establecidas en el artículo 16"2.

El paciente tiene un derecho a aceptar o rechazar cualquier tipo de tratamiento de salud que le concierna, con limitaciones que veremos más adelante. La ley agrega que el consentimiento debe ser ejercido en forma libre, voluntaria, expresa e informada ${ }^{2}$. Para que sea efectivo, la ley exige que el profesional proporcione al paciente información oportuna, adecuada, suficiente y comprensible $e^{2,3}$. Esta información debe abarcar su estado de salud, el posible diagnóstico de su enfermedad, alternativas de tratamiento disponibles y los riesgos que puedan representar, el pronóstico esperado y el proceso del postoperatorio previsible ${ }^{3}$. Si no fuere posible entregar esta información al paciente, debe 
ser facilitada a su representante legal o a la persona bajo cuyo cuidado se encuentre ${ }^{3}$.

En cuanto a las formalidades, el consentimiento se presta de manera verbal. Sin embargo, debe constar por escrito en la ficha clínica en los siguientes casos: intervenciones quirúrgicas, procedimientos diagnósticos y terapéuticos invasivos $y$, en general, en casos de procedimientos que impliquen un riesgo relevante y conocido para la salud del individuo ${ }^{4}$. Junto con ello, la ley presume que el paciente ha recibido la información pertinente cuando su firma conste en el documento que explique el procedimiento a que va a someterse ${ }^{4}$.

Ahora bien, la ley contempla tres hipótesis en las cuales se puede ejecutar procedimientos sin contar con la voluntad del paciente o su apoderado:

- Cuando la falta de intervención implique un riesgo para la salud pública.

- Cuando la intervención médica inmediata sea necesaria para prevenir riesgo vital o secuela funcional grave y el paciente no pueda manifestar su voluntad por su condición de salud.

- Cuando el paciente esté incapacitado para consentir ${ }^{5}$.

\subsection{La limitación al consentimiento informado}

El artículo 14 establece tres limitaciones a la voluntad del paciente:

"En ningún caso el rechazo a tratamientos podrá tener como objetivo la aceleración artificial de la muerte, la realización de prácticas eutanásicas o el auxilio al suicidio"4.

El derecho del paciente no incluye la aceleración artificial de la muerte, la eutanasia o el auxilio al suicidio. El problema es que la ley no define esas situaciones.

\section{El consentimiento informado en los enfermos terminales}

La ley dedica un acápite ${ }^{6}$ a los pacientes que estén en condición terminal. Estos pacientes tienen derecho a decidir y rechazar cualquier tratamiento que tenga como efecto prolongar artificialmente su vida ${ }^{7}$. La ley reitera la prohibición de acelerar artificialmente la muerte.

Esta regulación no es distinta de la que vimos en el artículo 14 pues el enfermo terminal tiene el mismo derecho que los demás pacientes. Además, el derecho a rechazar tratamientos que prolonguen artificialmente su vida (el denominado encarniza- miento terapéutico) se puede entender comprendido en el artículo 14. La ley indica que este derecho no afecta la mantención de las medidas de soporte ordinario aunque la ley no define cuáles son las medidas ordinarias y las extraordinarias.

Finalmente, la ley repite dos exigencias que están señaladas en normas precedentes: el deber de proporcionar información completa y comprensible, y la improcedencia del derecho a consentir cuando esté en riesgo la salud pública.

\section{El consentimiento informado y los Comités de Ética}

La ley ha previsto la intervención de Comités de Ética en una serie de casos, incluyendo aquellos en que el paciente ejerza su derecho de autonomía. Señala la ley que el profesional tratante debe solicitar la opinión del Comité cuando estime que la decisión del paciente lo “...expone a graves daños a su salud o al riesgo de morir, que serían evitables prudencialmente siguiendo los tratamientos indicados..." . También está previsto recurrir al Comité si el paciente rechaza "...la insistencia en la indicación de los tratamientos o la limitación del esfuerzo terapéutico..."

La intervención del Comité limita el derecho de autonomía del paciente pues, si este decide algo que expone su salud a consecuencias graves, el médico está obligado a consultar al Comité en vez de acceder a los requerimientos del paciente de inmediato.

La ley se encarga de precisar que el Comité de Ética no adopta decisiones, al señalar que su pronunciamiento reviste solamente el carácter de recomendación $n^{8}$. Además, la ley libera a los integrantes del Comité de toda responsabilidad civil o penal respecto de lo que eventualmente pudiere suceder al paciente ${ }^{8}$. Lo complicado es que la ley no dice nada sobre la responsabilidad del médico tratante.

Finalmente, la ley agrega que las personas que no estén de acuerdo con la opinión del Comité pueden recurrir a las Cortes de Apelaciones ejerciendo la Acción de Protección prevista en el artículo 20 de la Constitución ${ }^{8}$.

\section{Alta voluntaria y forzosa}

La ley dispone que el paciente tiene derecho a pedir el alta voluntaria cuando desee no ser tratado, quiera interrumpir el tratamiento o se niegue a cumplir las prescripciones médicas ${ }^{9}$. Como con- 
trapartida, la ley otorga al establecimiento de salud la facultad de decretar el alta forzosa del paciente ante las mismas circunstancias ${ }^{9}$.

La ley no entrega mayores precisiones acerca de este derecho del establecimiento de salud. Sólo indica que lo puede ejercer cuando el paciente desee no ser tratado, quiera interrumpir el tratamiento o se niegue a cumplir las prescripciones médicas. Por tanto, se produce aquí una situación que puede anular el derecho del paciente al consentimiento informado pues si el paciente se niega a un procedimiento, el establecimiento puede darlo de alta forzosamente.

\section{Objeción en conciencia y consentimiento informado}

La ley confiere al profesional de la salud un derecho de objeción en conciencia. Si el profesional no está de acuerdo con la decisión manifestada por el paciente, puede decidir no continuar como responsable del tratamiento ${ }^{8}$.

Este derecho se podría entender comprendido dentro del derecho constitucional que tienen todas las personas a la libertad de conciencia ${ }^{10}$. Sin embargo, eso podría ser objeto de disputa, de modo que resulta mejor que la ley lo reconozca expresamente.

Ahora bien, la ley se ha encargado de proteger al paciente. En efecto, la ley dispone que si el profesional desea ejercer este derecho, debe asegurar que el cuidado del paciente sea asumido por otro profesional calificado.

\section{La nueva normativa sobre el derecho del paciente al consentimiento informado y sus limitaciones}

1. El derecho al consentimiento informado es un derecho de autonomía. Esto queda claro del título del párrafo 60: "De la autonomía de las personas en su atención de salud." Como se ha visto, este derecho de autonomía se encuentra limitado de varias maneras:

a) La ley no permite acelerar artificialmente la muerte, la eutanasia ni el auxilio al suicidio.

b) Incluso en aquellas conductas que sí están permitidas por la ley, la voluntad del paciente puede quedar anulada en varios casos:

c) En casos graves es obligatoria la intervención del Comité de Ética, de modo que el médico no puede acceder de inmediato al requerimiento del paciente.

d) En casos menos graves, la intervención del Comité de Ética queda a discreción del médico ${ }^{8}$, de modo que también puede negarse a acceder a la voluntad del paciente.

e) El establecimiento de salud puede decretar alta forzosa en vez de someterse a la voluntad del paciente.

f) El derecho del médico a la objeción de conciencia también podría hipotéticamente funcionar como un límite a la libertad del paciente. El paciente podría inhibirse de ejercer su derecho si sabe que el médico puede renunciar a seguir tratándolo y él no quiere perderlo.

Por tanto, las situaciones señaladas permiten concluir que el paciente no tiene un derecho fuerte de autonomía que deba siempre ser respetado por el médico o el establecimiento de salud.

2. La ley tiene problemas de imprecisión respecto a los límites al derecho de autonomía.

a) La ley prohíbe la eutanasia pero no define ese concepto. El problema es que existe discusión sobre qué es eutanasia. Goic ${ }^{11}, \mathrm{Beca}^{12}, \mathrm{Ugarte}^{13}$, Rodríguez $^{14}$ y Vivanco ${ }^{15}$ sostienen que desconectar a un paciente de un ventilador mecánico no constituye eutanasia. En cambio, Foot ${ }^{16}$ y Gruzalski ${ }^{17}$ creen que es una forma de eutanasia pasiva y Hopkins ${ }^{18}$ defiende que es eutanasia activa pues desconectar es una acción.

b) El artículo 14 prohíbe acelerar artificialmente la muerte. Desconectar de un ventilador ¿puede considerarse una intervención (es decir, algo artificial) que acelera la muerte? Quizá algunos médicos estimen que no, pero los jueces pueden pensar que sí. En consecuencia, se puede generar un problema.

c) El artículo 14 prohíbe el auxilio al suicidio. Puede ser problemático identificar esta situación. Por ejemplo, si un paciente prefiere morir rechazando un tratamiento que lo salvaría y el Comité de Ética y su médico tratante lo respaldan, ¿no es eso ayudar al paciente a morirse?

La conclusión es que la ley no es precisa y los médicos pueden razonablemente pensar que la ley no aclara sus responsabilidades. Como vimos, sólo el Comité de Ética está libre de responsabilidad. 
3. La limitación a la autonomía del paciente y a su derecho al consentimiento informado puede explicarse porque la ley aparentemente asume la distinción entre matar y dejar morir y la doctrina del doble efecto, tal como lo hace numerosa doctrina nacional, según se ha demostrado en otra parte ${ }^{19}$. Es por eso que si el paciente lo desea, se permite desconectarlo pero no inyectarle una droga para matarlo aunque él lo pida (eutanasia). El primer caso se permite porque consiste en dejar morir en tanto el segundo se prohíbe porque consiste en matar. De igual manera, se permite hacer una cosa que pueda causar la muerte (inyectar sedación) pero no otra que tenga igual resultado (inyección de droga para matar: eutanasia), si la intención en el primer caso era aliviar el sufrimiento, bajo la doctrina del doble efecto.

El problema es que existe abundante literatura que cuestiona la validez de la distinción entre matar y dejar morir, como Rachels ${ }^{20}$, Foot ${ }^{16}$, Gruzalski $^{17}$, Hopkins ${ }^{18}$ y Dworkin ${ }^{21}$ y también la utilidad de la doctrina del doble efecto, como Foot ${ }^{22}$, Thomson $^{23}$, Davis ${ }^{24}$, McIntyre ${ }^{25}$, Scanlon ${ }^{26}$, Hart $^{27}$ y Kamm ${ }^{28}$. Estos argumentos se han desarrollado en otra parte ${ }^{19}$, de modo que no cabe repetirlos.

Por tanto, es posible pensar que la nueva ley limita severamente la autonomía de los pacientes sobre bases que puedan estar equivocadas o, al menos, ser objetables.

4. Puede estimarse que la ley falla en un doble sentido. Por una parte, porque limita la autonomía de las personas de una manera que pueda estimarse, al menos, discutible. Por otra, porque no regula un aspecto crítico de la práctica de la medicina que consiste en que, en ocasiones, los médicos deben adoptar decisiones de vida y muerte. Como hemos visto, la ley no permite tomar estas decisiones.

Por tanto, parece que la ley no está muy clara y decidida sobre cómo regular la disponibilidad de la vida humana. ¿A qué se puede deber esto? Es posible que en nuestra idiosincrasia no tengamos resuelto sobre los casos en que podemos disponer de la vida humana. Esto se puede advertir en los sucesivos cambios que experimentó esta ley durante su tramitación. Esos cambios revelan que no había posturas claras y dominantes. De hecho, algunos de esos cambios fueron súbitos y descuidados. Un ejemplo es el testamento vital. Esta institución estaba prevista en el proyecto de ley en el artículo 18. A último minuto, sin explicación, se eliminó el testamento vital, pero esa sección de la ley se sigue llamando "Del estado de salud terminal y la voluntad manifestada previamente" aunque la voluntad manifestada previamente ya no está regulada por la ley.

Estas consideraciones permiten pensar que es posible que esta ley sea objeto de revisiones en algún futuro cercano.

\section{Conclusiones}

1. El paciente tiene derecho a un consentimiento informado, que le permite rechazar cualquier tratamiento médico. Para ejercer este derecho, el paciente debe recibir información completa y oportuna. Sin embargo, el paciente no tiene derecho a acelerar artificialmente la muerte, exigir eutanasia o el auxilio al suicidio.

2. El paciente terminal tiene derecho a oponerse a medidas que prolonguen artificialmente su vida. Se le aplican las limitaciones antes señaladas.

3. Está prevista la intervención de un Comité de Ética cuando el médico estime que la decisión manifestada por el paciente lo expone a graves daños a su salud o al riesgo de morir, que serían evitables prudencialmente, o cuando el paciente rechace la insistencia en la indicación de los tratamientos o la limitación del esfuerzo terapéutico.

4. El médico tiene derecho a la objeción en conciencia frente a los requerimientos del paciente.

5. El paciente tiene derecho a solicitar el alta voluntaria en caso de que no esté de acuerdo con el equipo médico $y$, por su parte, el establecimiento de salud también tiene la facultad de decretar el alta forzosa del paciente si este no desea cumplir las prescripciones médicas.

6. La ley establece expresamente que se prohíbe la aceleración artificial de la muerte, la realización de prácticas eutanásicas o el auxilio al suicidio. El problema es que esta ley no define esas hipótesis, de modo que podría estimarse, por ejemplo, que la desconexión de un ventilador mecánico constituye una conducta prohibida.

7. Las diversas limitaciones que la ley pone a la autonomía del paciente se podrían explicar porque la ley parece asumir la distinción entre matar y dejar morir y la doctrina del doble efecto, en circunstancias que existe abundante literatura que cuestiona la validez de ambas. 
8. La ley no regula una parte de la práctica médica, que consiste en adoptar decisiones de vida y muerte. Es posible que esto ocurra porque no exista acuerdo en nuestro medio sobre cuando disponer de la vida de las personas. Esto se puede apreciar en los diversos y repentinos cambios que sufrió esta ley durante su tramitación.

\section{Referencias}

1. Artículo transitorio. Ley $\mathrm{N}^{\circ}$ 20.584. Diario Oficial, República de Chile.

2. Artículo 14.

3. Artículo 10.

4. Artículo 14.

5. Artículo 15.

6. Parágrafo 2. "Del estado de salud terminal y la voluntad manifestada previamente."

7. Artículo 16.

8. Artículo 17.

9. Artículo 18.

10. Artículo $19 \mathrm{n}^{\circ} 6$ de la Constitución.

11. Goic A. Apuntes sobre la Eutanasia. Rev Med Chile 2005; 133: 371-5.

12. Beca JP, Ortiz A, Solar S. Derecho a Morir. Rev Med Chile 2005; 133: 601-6.

13. Ugarte JJ. El derecho a la vida y la Constitución. Rev Chil Derecho 2006; 33: 509-27.
14. Rodríguez E. La eutanasia y sus argumentos. Ars Medica 2000; 2: 45-57.

15. Vivanco A. La eutanasia ante el derecho. Ars Medica 2006; 12: 53-92.

16. Foot P. Euthanasia. Philos Public Aff 1977; 6: 85-112.

17. Gruzalski B. Killing by Letting Die. Mind 1981; 90: 91-8.

18. Hopkins PD. Why does removing machines count as "passive" euthanasia? Hastings Cent Rep 1997; 27: 29-37.

19. Figueroa R. Matar, dejar morir y eutanasia en el proyecto de ley sobre derechos de las personas y en la doctrina chilena. Rev Med Chile 2011; 139: 655-9.

20. Rachels J. Active and Passive Euthanasia. N Engl J Med 1975; 292: 78-80.

21. Dworkin. G. Sex, Suicide, and Doctors, Ethics 1999; 109: 579-85.

22. Foot P. The Problem of Abortion and the Doctrine of the Double Effect. Oxford Rev 1967; 5: 5-15.

23. Thomson JJ. Physician-Assisted Suicide: Two Moral Arguments. Ethics 1999; 109: 497-518.

24. Davis N. The Doctrine of Double Effect: Problems of Interpretation. Pac Philos Quart 1984; 65: 107-23.

25. McIntyre A. Doing Away with Double Effect, Ethics 2001; 111: 219-55.

26. Scanlon T. Moral Dimensions. Belknap Press of Harvard University Press England; 2008.

27. Hart HL A. Punishment and Responsibility: Essays in the Philosophy of Law. Oxford University Press USA; 2008.

28. Kamm, F.M The Doctrine of Double Effect: Reflexions on Theoretical and Practical Issues. J Med Philos 1991; 16: 571-85. 